\title{
A Tip-Tilt Mirror Control System for Partial Image Correction at UC Mount John Observatory
}

\author{
Jiayu Liu ${ }^{1, *}$, Vishnu Anand Muruganandan ${ }^{1}$, Richard Clare ${ }^{1}$, María Cruz Ramírez Trujillo ${ }^{2}$, Stephen J. Weddell ${ }^{1}$ \\ ${ }^{1}$ Department of Electrical and Computer Engineering; University of Canterbury, Christchurch, New Zealand \\ ${ }^{2}$ Instituto de Astrofísica de Canarias (IAC): La Laguna, Tenerife, Spain \\ *Corresponding Author: jake.liu@pg.canterbury.ac.nz
}

\begin{abstract}
Astronomical images captured by ground-based telescopes, including at University of Canterbury Mount John Observatory, are distorted due to atmospheric turbulence. The major constituents of atmospheric distortion are tip-tilt aberrations. The solution to achieve higher resolution is to develop and install a tip-tilt mirror control system on ground-based telescopes. A realtime tip-tilt mirror control system measures and corrects for tip-tilt aberrations in optical wavefronts. It effectively minimises the perturbation of the star image when observing with the aid of a telescope. To the best of our knowledge, this is the first tip-tilt mirror control system to be applied at a New Zealand astronomical observatory. This would extend the possibilities of correcting higher-order aberrations for 0.5 to 1.0 metre class, ground-based telescopes.

Index Terms-image correction, tilt sensing, wave-front sensing, adaptive optics
\end{abstract}

\section{INTRODUCTION}

Adaptive optics (AO) is a real-time closed-loop system that can measure and correct perturbed incoming wavefronts at the telescope and can produce high resolution images [1]. Tip and tilt are low order aberrations, and these aberrations are the results of deviation of light propagated in both $\mathrm{x}$ and $\mathrm{y}$ directions. [2] [3]. Therefore, tip and tilt correction can be very efficient for solving what one might consider to be the effect of blurring as it can reduce $87 \%$ of the total aberration [4].

The correction is performed by controlling the tip-tilt mirror in two axes. The mirror deflects in the range of micrometers and controls the optical path in two axes to compensate for deviations in the wavefront. This paper describes our current research on the tip-tilt mirror imaging and control system that can measure and correct tip and tilt image distortions in realtime.

Currently, we are in the testing phase of our prototype system. This prototype is built for the Boller and Chivens (BC) 61-cm f/6.25 telescope at Mt. John Observatory. Our target frequency for tip-tilt correction is $50 \mathrm{~Hz}$. This frequency is based on the turbulence profiles of Mt. John Observatory [5].

Section II of this paper introduces a set of functions used to represent optical aberrations, i.e., tip-tilt representations, in terms of Zernike polynomials. In addition, tilt wavefront sensing and image threshold algorithms are explained. Section III presents a tip-tilt control system and equipment that has been used for our system. Section IV discusses the control voltage response and presents results from our closed-loop testing. Finally, other factors and future development are discussed in Section V.

\section{BACKGROUND}

Optical wavefronts from an astronomical object are distorted by Earth's atmosphere due to phase changes over time. Therefore, a long-exposure focused image is perturbed on the focal plane of a ground-based telescope during observations of astronomical objects.

In this section, Part A reviews some mathematical descriptions and characteristics of the tilt aberration. Part B describes the methods used for thresholding. Part $\mathrm{C}$ introduces algorithms of star centroid estimation. Part D provides a comparison of centroiding accuracy improvements performed by thresholding and Weighted Centre of Gravity (WCG).

\section{A. Zernike Polynomials and Tilt}

Zernike polynomials are a set of orthonormal basis functions which have been used to describe optical system aberrations. Zernike polynomials, which are described in [2], are a set of functions orthogonal over the unit circle, and they are usually described in terms of normalised polar coordinates as $Z_{n}^{m}(\rho, \theta)$, where $n$ describes the power of the radial polynomial, $m$ describes the angular frequency, $\rho$ is the radius in the image phase in the normalised plane, and $\theta$ is the angle between image phase and $x$ axis.

Tilt aberration is the first order of Zernike Polynomials and comprise 87 percent of the energy from a wavefront aberration [4]. For tilt in the $y$-direction, $n$ equals 1 and $m$ equals -1 . For tilt in $x$-direction, $n$ equals 1 and $m$ equals 1 . The equations are shown in Eq.1,

$$
\begin{aligned}
& y: Z_{n}^{m}(\rho, \theta)=2 \rho \sin (\theta), \\
& x: Z_{n}^{m}(\rho, \theta)=2 \rho \cos (\theta) .
\end{aligned}
$$

Each order of polynomials represent different types of aberrations such as tip, tilt, spherical aberration, astigmatism, and coma [2].

The image intensity distribution of the tilt aberration is the same as the unshifted image. This has been proved by simulation of the Point Spread Function (PSF) and Modulation Transfer Function (MTF) [2]. The PSF is a description of a normalised intensity distribution of the point source image. 
PSF and MTF representations of wave aberration corresponding to each Zernike mode shows a result where the tilt modes $(n=1 ; m=1,-1)$ are linear phase slopes across the pupil, and often result in an $x$ and $y$ phase shift without changing the shape of the PSF. Therefore, at the image plane, the PSF is shifted in a direction of tilt and is perpendicular to the direction of wavefront propagation. The distance of the shift is proportional to the amount of tilt. The tip and tilt aberrations are demonstrated in Figure 1. The PSF of two consecutive frames of a point source is plotted in the same figure. The difference of $\left(x_{i}-x_{0}\right)$ and $\left(y_{i}-y_{0}\right)$ represent the tip and tilt aberrations in the spatial domain.

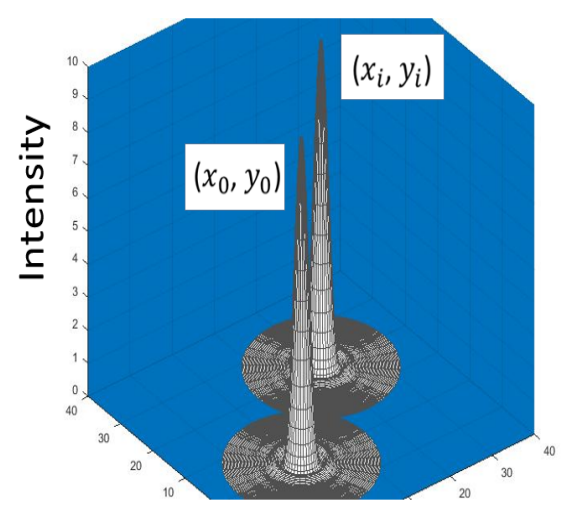

Figure 1. Example of tip-tilt aberration.

Figure 2 shows the geometric relationships of wavefront tilt and resulting displacement on a focused image [6]. The geometry illustrates that if a beam with a tilt $K$ enters the aperture of radius $r$ and propagates over distance $R$, the angle between the propagation and optical axis is $\tan \left[\frac{K}{r}\right]$ and the centroid of the image would be shifted by $\left(\frac{R}{r}\right) K$.

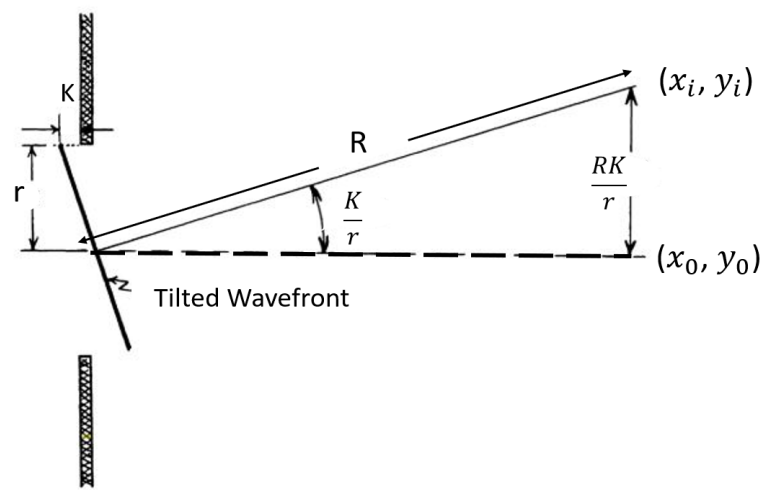

Figure 2. Displacement of the centre of a beam due to wavefront tilt of magnitude K. [6]

The perturbations result is two centroid terms $(\Delta x, \Delta y)$ from a natural star, which are proportional to the tilt of the star's wavefront at the time of capture and over the period of which its displacement is estimated. Thus, we consider the correction of centroid displacement as the solution of the tilt aberration in both $x$ and $y$ directions at the image plane. The relationships between the phase of tilt and the displacement of the image centroid is:

$$
\tan \frac{K_{i}}{r_{0}}=\frac{\left(\Delta x_{i}, \Delta y_{i}\right)}{f},
$$

where $r_{0}$ is the sub-aperture radius and $f$ is the focal length of the lenslet array, and $K_{i}$ is the local tilt of the wavefront.

\section{B. Thresholding}

We consider three algorithms for thresholding the detected image $I_{i, j}$. These thresholding algorithms are, the Global Average (GA), Ostu, and Iterative Threshold (IT). The GA algorithm is the simplest method, which calculates the average value of the image [7]. The equation is:

$$
T=\frac{\sum_{i, j} I_{i, j}}{i_{\max } j_{\max }}
$$

where $i, j$ are pixel positions and $i_{\max }, j_{\max }$ are the dimensions of the image.

The second algorithm, Ostu, calculates a threshold T, which can maximise the variance between foreground and background. A weighted sum of the variance is defined as [8]:

$$
\sigma_{\omega}^{2}(t)=\omega_{f}(t) \sigma_{f}^{2}(t)+\omega_{b}(t) \sigma_{b}^{2}(t),
$$

where the $\omega_{f}(t), \omega_{b}(t)$ are the probabilities of the foreground and background separated by threshold value $\mathrm{t}$, and $\sigma_{f}^{2}(t)$, $\sigma_{b}^{2}(t)$ are variances of foreground and background. For foreground and background, the weighted variance $\sigma_{\omega}^{2}(t)$ needs to be minimised, therefore, the aim is to find a threshold $t$ that can maximise the variance between both [8]:

$$
\begin{aligned}
\sigma_{b}^{2}(t) & =\sigma^{2}-\sigma_{f}^{2}(t) \\
& =\omega_{f}\left(\mu_{f}-\mu_{T}\right)^{2}-\omega_{b}\left(\mu_{b}-\mu_{T}\right)^{2},
\end{aligned}
$$

where $\mu_{f}, \mu_{b}$ are the mean of the foreground and background.

The Ostu algorithm is limited however, in that this method is only effective for an image if its histogram has a bimodal distribution. However, for the single star object, the performance of this algorithm decreases since the histogram no longer exhibits bimodality [9].

The Iterative Threshold algorithm that we employ and that is described here, is effective for images where the histogram is monomodal [7]. The process starts by averaging the maximum and minimum intensity values:

$$
T^{0}=\frac{I_{\min }+I_{\max }}{2},
$$

where the initial threshold is $T^{0}$. According to $T^{0}$, the image can be divided into foreground and background. The average intensity values of foreground and background are $A_{f}$ and $A_{b}$ :

$$
\begin{array}{r}
A_{f}=\frac{\sum_{I=T_{0}+1}^{I} \times \operatorname{hist}(\mathrm{I})}{\sum_{I=T_{0}+1} \operatorname{hist}(\mathrm{I})} \\
A_{b}=\frac{\sum_{I}^{T_{0}} I \times \operatorname{hist}(\mathrm{I})}{\sum_{I}^{T_{0}} \operatorname{hist}(\mathrm{I})},
\end{array}
$$


then,

$$
T^{k}=\frac{A_{b}+A_{f}}{2} .
$$

If $T^{k}=T^{k+1}$, the iteration is stopped and $T^{k}$ is the final threshold value.

\section{Centriod Estimation}

The accuracy of the centroid estimation can be improved by both equipment setup and software algorithms. A typical equipment setup is to make the star sensor slightly de-focused so that the size of a star spot on the image sensor is not smaller than $3 \times 3$ pixels. [10]. Therefore, a star spot conforms to an "Airy disk", and its intensity can be approximated by the Gaussian function so that centroids of subpixel displacement on the wavefront sensor are more accurate [11].

The Centre of Gravity (COG) is the most commonly used algorithm for centroiding [12]. The formula of COG is:

$$
\begin{aligned}
& x_{c}=\frac{\sum_{i, j} x_{i} I_{i, j}}{\sum_{i, j} I_{i, j}}, \\
& y_{c}=\frac{\sum_{i, j} y_{j} I_{i, j}}{\sum_{i, j} I_{i, j}},
\end{aligned}
$$

where $I_{i, j}$ is the intensity of the light on the detector pixels and $x_{i}, y_{j}$ are weighting numbers that related to the particular position of pixel $(i, j)$.

The first method to improve the accuracy of COG is to apply threshold $\mathrm{T}$ to the noise image $I_{i, j}$, which subtracts a threshold $\mathrm{T}$ from the background noise, and zeros negative pixels. The formula of this method COG is:

$$
\begin{aligned}
& x_{c}=\frac{\sum_{i, j} x_{i}\left(I_{i, j}-T\right)}{\sum_{i, j}\left(I_{i, j}-T\right)}, \\
& y_{c}=\frac{\sum_{i, j} y_{j}\left(I_{i, j}-T\right)}{\sum_{i, j}\left(I_{i, j}-T\right)} .
\end{aligned}
$$

An alternative method to improve the accuracy of COG is the Weighted Centre of Gravity (WCOG). This method is a variation of COG, and weight values $W_{i, j}$ are applied to the target image $I_{i, j}$ [10]. The weight values are theoretically assigned by the Gaussian function, however, the calculations are complex and time consuming. Alternatively, we employed a practically equivalent weighting method. Instead of applying a Gaussian to fit the target image, the weight values $W_{i, j}$ are replace by a previously generated reference image $R_{i, j}$, and this reference image $R_{i, j}$ is Gaussian distributed [13]. An example of reference image $R_{i, j}$ is shown in Figure 3. Therefore, the centroid estimate by WCOG is then:

$$
\begin{aligned}
& x_{c}=\frac{\sum_{i, j} x_{i} I_{i, j} R_{i, j}}{\sum_{i, j} I_{i, j} R_{i, j}}, \\
& y_{c}=\frac{\sum_{i, j} y_{j} I_{i, j} R_{i, j}}{\sum_{i, j} I_{i, j} R_{i, j}} .
\end{aligned}
$$

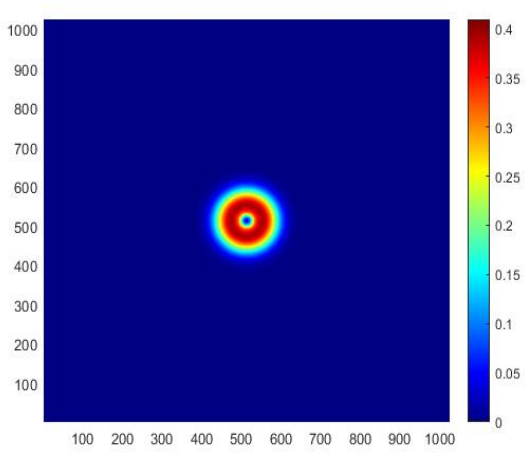

Figure 3. A reference image $R_{i, j}$ for WCOG.

\section{Algorithm Evaluation}

To evaluate the effectiveness of centroiding methods in this section, we compare three algorithms COG, COG with Iterative Threshold (COGIT) and WCOG by using a stationary picostar device ${ }^{1}$. Table I shows centroid results $\left(x_{c}, y_{c}\right)$ by using COG, COGIT and WCOG, and the variance of $x_{c}$ and $y_{c}$ from results of these three centroiding algorithms.

TABLE I: Results of the centroid $\left(x_{c}, y_{c}\right)$ and the variance of results by using COG, COG IT and WCOG.

\begin{tabular}{l|l|l|l|l}
\multirow{2}{*}{ Algorithm } & \multicolumn{2}{|c|}{ Centroid Coordinate (pixels) } & \multicolumn{2}{c}{ Variance (pixels) ${ }^{2}$} \\
\cline { 2 - 3 } & $x_{c}$ & $y_{c}$ & $\sigma_{x_{c}}^{2}$ & $\sigma_{y_{c}}^{2}$ \\
\hline COG & 207.1763 & 53.0536 & \multirow{2}{*}{0.0032} & \multirow{2}{*}{2.7526} \\
\hline COGIT & 207.2657 & 50.6756 & &
\end{tabular}

To view the visual effect of the WCOG and COGIT, an image of the TDRS-11 satellite was processed by both the Iterative Threshold (IT) algorithm and WCOG. Figure 4 shows a comparison of accuracy improvements by these two algorithms. Although the centroid estimation is the same, Subfigure 4(a) preserves more aberration details, which may be used by a wavefront sensorless algorithm, for example [14], where higher order aberrations may be removed.

\section{PROPOSED METHOD}

In this section, we propose our solution of tip-tilt correction. Figure 5 shows a layout of a laboratory setup. The light source is a collimated laser beam. Tip aberrations are generated and applied to the laser beam by a Spatial Light Modulator (SLM). These devices can modulate light spatially in amplitude and phase, so they act as a dynamic optical element [15]. The sensing camera iXon [16] calculates the centroid shifts in real-time, and transfers the centroid coordinates to the PID controller. The controller then calculates the corresponding correction voltage for the tip-tilt piezoelectric mirror. The horizontal and vertical motions are controlled by separate voltage channels. The tip-tilt mirror (TTM) requires the mirror

\footnotetext{
${ }^{1}$ https://astromart.com/classifieds/astromart-classifieds/misc-otherastronomy/show/picostar-artificial-star-for-collimation
} 


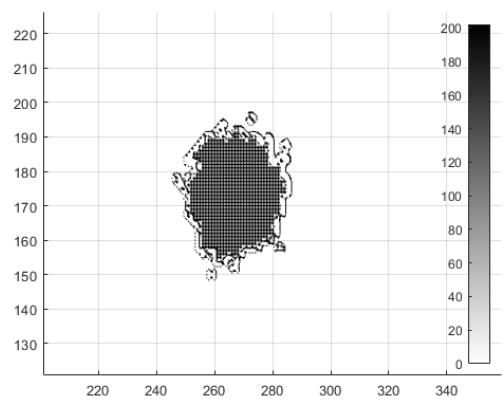

(a)

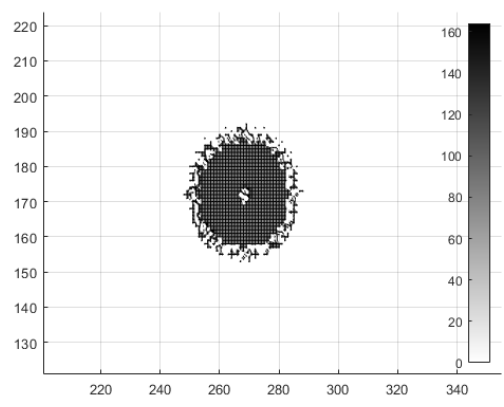

(b)

Figure 4. A comparison of image intensity by using the Iterative Threshold (IT) and WCOG algorithms, where the inverse of both Subimages are shown to provide more detail. (a) Image of TDRS-11 processed by the IT method. (b) Image of TDRS-11 processed by the WCOG method.

driver to control its piezoelectric actuator. The mirror driver can be considered as a high-voltage amplifier. It takes the control voltage of the PID controller at the low voltage (LV) input and amplifies to 1000 Volts for driving the tip-tilt mirror at high voltage (HV) output.

To improve the convenience of our AO operation system, a graphical user interface (GUI) was developed for our iXon camera. Figure 6 shows the GUI of using the iXon 897 camera of an image of Sirius, taken at 04:30 NZST on Sunday 13 September from Mt. John Observatory. Apart from the standard operations, such as triggering and binning, this software also includes the centroid sensing algorithm and communications with the PID controller.

For tip-tilt correction, the centroid algorithm will generate an inverse response to the tip-tilt aberration, and performance can be determined through the design of a controller. By conducting an instrument calibration, the correction voltage and tip/tilt displacements were found to have a linear relationship. The relationship between per-pixel and voltage on both $\mathrm{x}$ and y channels are:

$$
\begin{gathered}
\Delta x=0.138 \times \Delta V_{x} \\
\Delta y=0.178 \times \Delta V_{y},
\end{gathered}
$$

where $\Delta x$ and $\Delta x$ represents the centroid $(x, y)$ displace-

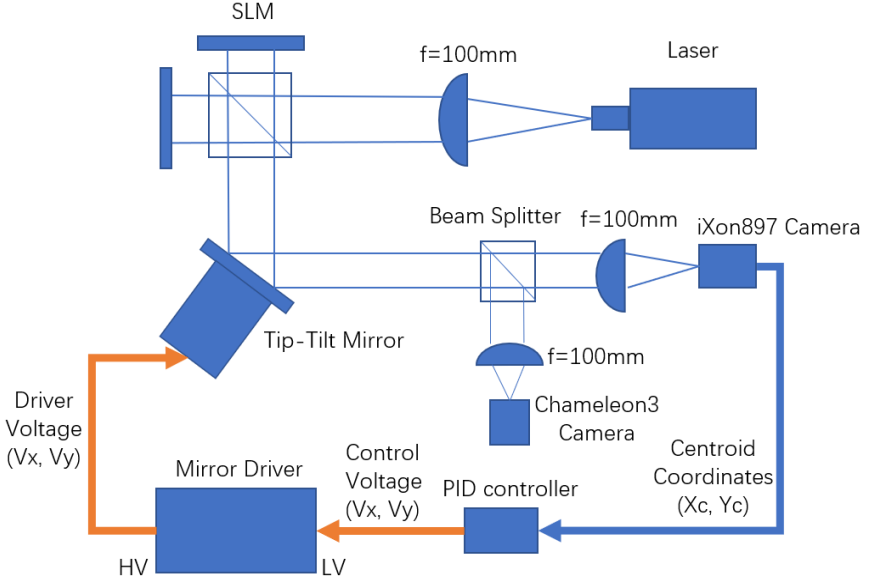

Figure 5. Laboratory layout of tip-tilt mirror control system.

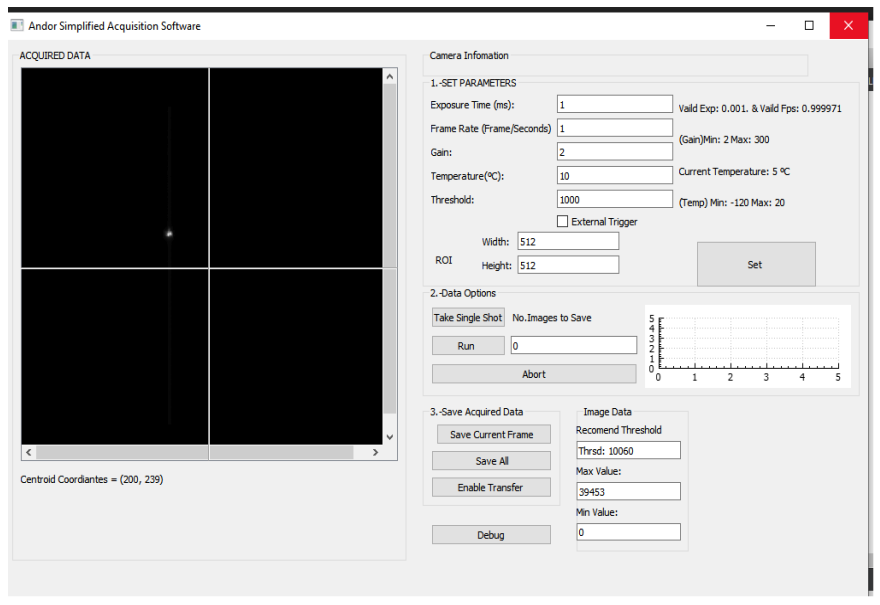

Figure 6. The GUI of the iXon camera sensor for tip-tilt correction

ments, $\Delta V_{x}$ and $\Delta V_{y}$ represents the change of control voltage $\left(V_{x}, V_{y}\right)$. Hence, a PID controller was implemented for the tiptilt mirror driver based on [17],

$$
\Delta c(t)=K_{p} e(t)+K_{d} \frac{d e(t)}{d t}+K_{i} \int_{0}^{t} e(t) d t
$$

where, $K_{p}, K_{i}$ and $K_{d}$ are proportional, integral and derivative gains and $e(t)$ is the error between the reference centroid and centroid shifts, and $\Delta c(t)$ is the compensation for centroid shit.

Due to the lack of characteristics of the tip-tilt mirror driver, the controller gains were calculated by trial and error by monitoring the motion of the image centroid. Each gain value on both tip and tilt control channels is listed in Table II. 
TABLE II: Gains of tip and tilt controllers.

\begin{tabular}{l|c|r} 
Gains & Tip & Tilt \\
\hline$K_{p}$ & 0.400 & 0.400 \\
$K_{i}$ & 0.023 & 0.020 \\
$K_{d}$ & 0.250 & 0.280
\end{tabular}

\section{RESUlts AND Discussion}

\section{A. Control Voltage Response}

In this section, we describe the measurement of open-loop voltage responses for the PID controller. A simulation of tilt aberrations, in terms of centroid shifts, $\Delta x$ was generated for the PID controller. Figure 7 shows a plot of the tilt aberrations as a function of centroid shifts $f(x)$ and the corresponding calculated correction voltage.

$$
\Delta x=0.138 \times(\text { Tilt.setpoint }-f(x))
$$

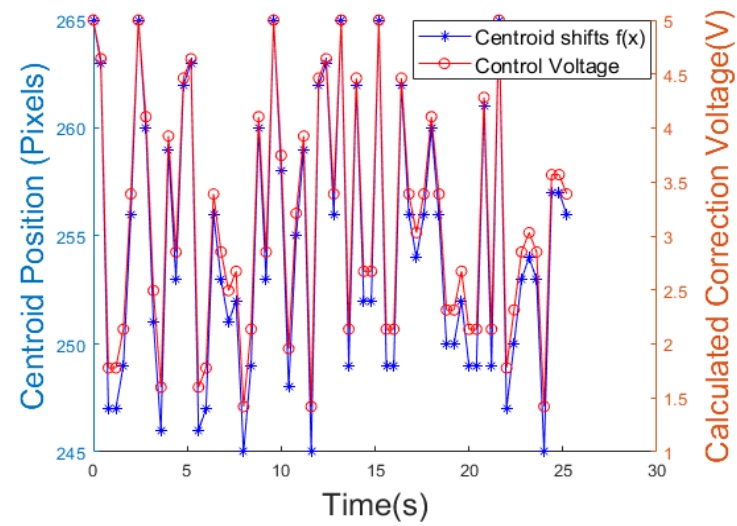

Figure 7. Measurement of centroid shift and desired correction voltage on $\mathrm{x}$-axis.

Over a series of comparisons, the correction voltage generated by the PID controller was measured, such as shown in Subfigure 8 (a). The simulated results of Figure 7 are identical to the trace of the oscilloscope actual measurements shown in Subfigure 8 (a) which were generated by the PID controller and are identical to the calculated voltage. Therefore, the proportional gain $K_{p}$ of our PID controller is accurate.

The gains of $K_{i}$ and $K_{d}$ show the settling time of our PID controller. Subfigure 8(b) shows the response time of the control system. The observed response time takes $14 \mathrm{~ms}$. Considering that the time taken for centroid processing is $34 \mathrm{~ms}$, the minimum response time is $48 \mathrm{~ms}$. Therefore, the maximum frequency of our current closed-loop controller is $20 \mathrm{~Hz}$.

\section{B. Measurement of close-loop tip-tilt mirror control}

We simulated our closed-loop tip-tilt mirror correction system before testing in the laboratory. The tip-tilt aberration is generated at the frequency of $2 \mathrm{~Hz}$, and the frame rate of the sensing camera was at 10 frames per second.

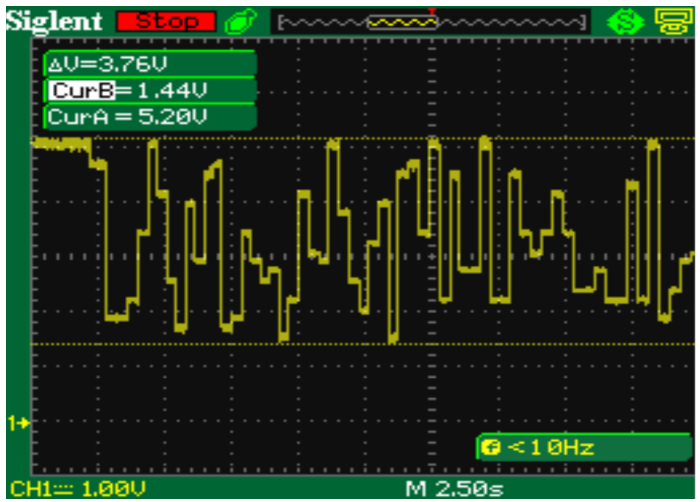

(a)

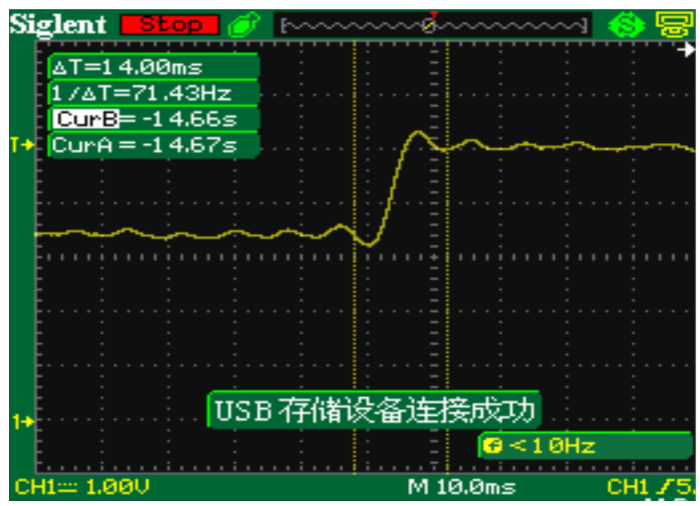

(b)

Figure 8. Control response measurements of tip-tilt aberrations in laboratory. (a) Correcting voltage measurement by oscilloscope. (b) Settling time of the PID controller.

The result of the x-axis correction is shown in Figure 9 . In Figure 9, where the red dotted line represents the centroid coordinates on the $\mathrm{x}$-axis, and the blue asterisks line represents the correction voltages. In the experiment, the centroid was shifted along both directions of the x-axis. The reference centroid coordinate is $x_{c}=261$; the red dots which are distributed near the reference centroid line represent the corrections of the tip-tilt mirror. The red dots which are distributed at triangular peaks represent that the simulated tilt aberration exceeds the maximum tilt angle of the tip-tilt mirror, and the tip-tilt mirror cannot correct such large tilt displacements.

Lastly, an instrument calibration test was conducted on the tilt angle of the tip-tilt mirror, and this was tested over a range of frequencies. Figure 10 presents the results of each frequency. As shown in the figure, the tilt angle of the tip-tilt mirror decreases when the frequency of the control voltage increases. These characteristics of our tip-tilt mirror were determined in the laboratory to ensure correction was performed over the required frequency range, and most importantly, before on-sky testing.

\section{CONCLUSION AND FUtuRE WORK}

In conclusion, we have designed and built an adaptive optics prototype for tip-tilt closed-loop control. This system has been 


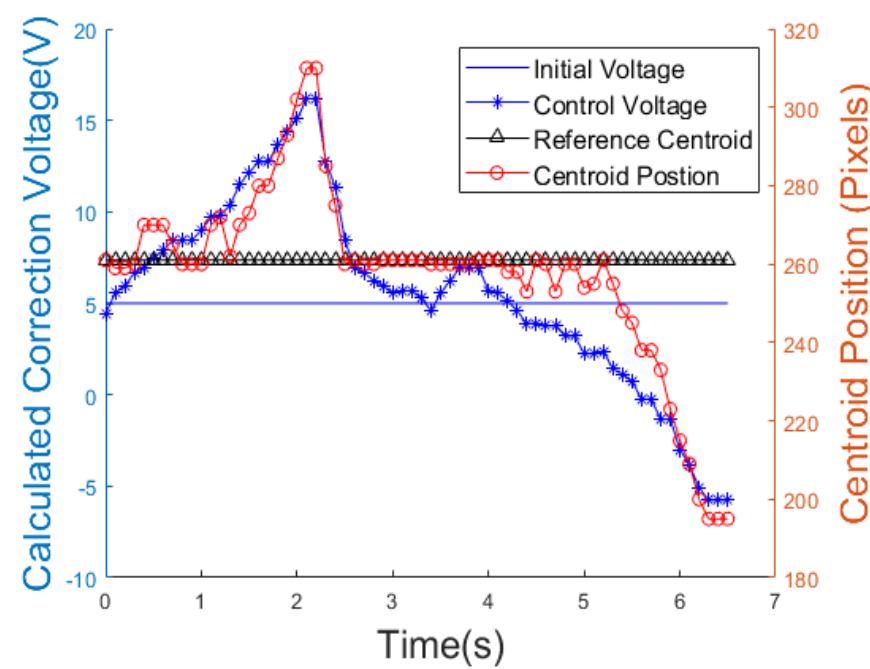

Figure 9. Measurement of closed-loop tip-tilt mirror on x-axis.

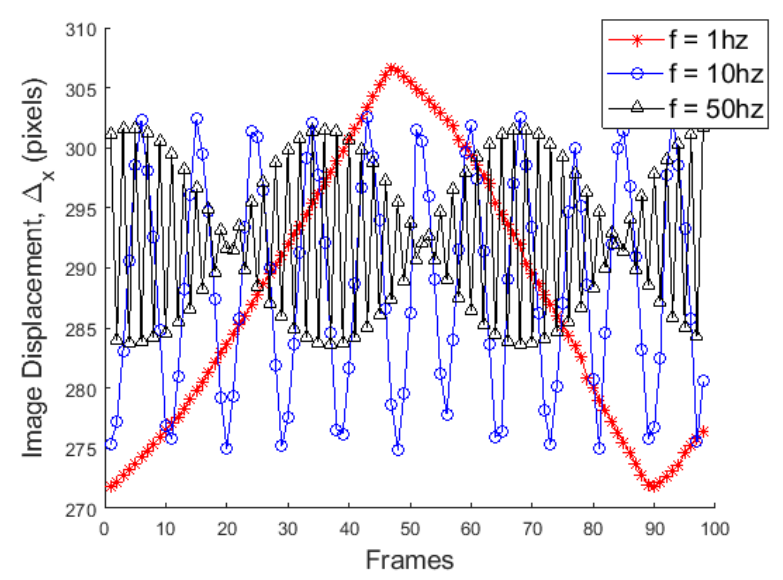

Figure 10. Frequency response of Tip-Tilt Mirror.

tested in the laboratory. Tests have shown that the system is able to correct tip and tilt at frequency of $2 \mathrm{~Hz}$. However, the laboratory test results also show that the tip-tilt boundary of the tip-tilt mirror system can satisfy an interim on-sky target of $20 \mathrm{~Hz}$.

The next step of our work is on-sky testing. Optimising the image processing algorithm is also an important requirement, and this will be done during our extended software development. Currently, the time taken for centroiding, communications and control response is $48 \mathrm{~ms}$. Further work will be required to optimise our centroiding routine and minimise current USB communication lag to achieve a realtime performance of $50 \mathrm{~Hz}$.

\section{ACKNOWLEDGMENT}

This project is supported by the Marsden Fund Council from Government funding, administered by the Royal Society of New Zealand (Marsden Fund Contract MFP- UOC1803).

The authors thank the University of Canterbury Mt. John Observatory for use of their facilities and the acquisition of Image data using the $61 \mathrm{~cm}$ Boller and Chivens telescope.

\section{REFERENCES}

[1] E. Fedrigo, R. Muradore, and D. Zilio, "High performance adaptive optics system with fine tip/tilt control," Control Engineering Practice, vol. 17, no. 1, pp. 122-135, 2009

[2] P. Y. Maeda, "Zernike polynomials and their use in describing the wavefront aberrations of the human eye."

[3] J. Watson, "Tip-tilt correction for astronomical telescopes using adaptive control." IEEE, 1997, pp. 490-494.

[4] M. C. Roggemann and B. Welsh, Imaging through turbulence. CRC Press, 1996.

[5] J. L. Mohr, "Atmospheric turbulence characterisation using scintillation detection and ranging," Ph.D. dissertation, 112009.

[6] R. K. Tyson, Principles of adaptive optics. Academic Press, 1991.

[7] Y. Xie, L. Li, H. Wang, and X. Zhao, "The application of threshold methods for image segmentation in oasis vegetation extraction," 062010 , pp. $1-4$.

[8] N. Otsu, "A threshold selection method from gray-level histograms," IEEE Transactions on Systems, Man, and Cybernetics, vol. 9, no. 1, pp. 62-66, 1979.

[9] J. Kittler and J. Illingworth, "On threshold selection using clustering criteria," IEEE Transactions on Systems, Man, and Cybernetics, vol. SMC-15, no. 5, pp. 652-655, 1985.

[10] X. Wan, G. Wang, X. Wei, J. Li, and G. Zhang, "Star centroiding based on fast gaussian fitting for star sensors," Sensors (Basel, Switzerland), vol. 18, 2018.

[11] B. Zhang, J. Zerubia, and J.-C. Olivo-Marin, "Gaussian approximations of fluorescence microscope point-spread function models," Appl. Opt., vol. 46, no. 10, pp. 1819-1829, Apr 2007.

[12] H. Li, H. Song, X. Rao, and C. Rao, "Accuracy analysis of centroid calculated by a modified center detection algorithm for shack-hartmann wavefront sensor," Optics Communications, 2008

[13] R. Clare, S. Weddell, and M. Louarn, "Centroiding of truncated shackhartmann laser guide star images with known reference images," 12 2019, pp. 1-6.

[14] M. J. Booth, "Wavefront sensorless adaptive optics for large aberrations," Opt. Lett., vol. 32, no. 1, pp. 5-7, Jan 2007.

[15] SLM Spatial Light Modulators, HOLOEYE Photonics AG.

[16] iXon Ultra 897, ANDOR-Oxford Instrunments, 52015.

[17] S. Reddy, R. Banyal, S. Rengaswamy, P. Kamath, and A. Selvaraj, "Development of image motion compensation system for $1.3 \mathrm{~m}$ telescope at vainu bappu observatory," 082019. 